\title{
LAS SOLUCIONES SUBESTRUCTURALES A LAS PARADOJAS Y EL PROBLEMA DE LA DEPENDENCIA
}

\author{
The Substructural Solution to Paradoxes \\ and the Problem of the Dependence
}

\author{
BRUNO DA RÉ ${ }^{a, b}$ \\ https://orcid.org/0000-0002-2958-7840 \\ brunohoraciodare@gmail.com
}

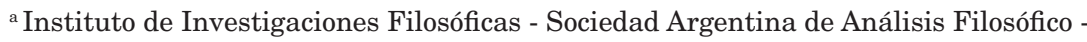
Consejo Nacional de Investigaciones Científicas y Técnicas, Buenos Aires, Argentina ${ }^{b}$ Universidad de Buenos Aires, Argentina

\section{Resumen}

En los últimos años se han desarrollado diversas soluciones subestructurales a las paradojas semánticas. En particular, se han postulado teorías no transitivas, no contractivas, no reflexivas y, recientemente, no monotónicas. Sin embargo, cuando dichas soluciones son presentadas mediante cálculos de secuentes surge el problema de la dependencia. En pocas palabras, este problema consiste en que no es posible separar las reglas estructurales de la formulación de las otras reglas del cálculo. En este artículo, presentaré este problema y mostraré que, de hecho, es posible construir un cálculo que no contiene ninguna regla estructural de manera explícita y que, sin embargo, resulta trivial al agregarle un predicado veritativo transparente con ciertos axiomas. Luego, delimitaré los alcances de dicho problema, concluyendo que la metodología correcta para seleccionar una solución subestructural a las paradojas semánticas debe basarse en argumentos filosóficos y, tal vez, en un estudio empírico sobre el fenómeno de la paradojicidad y no en la comparación de derivaciones en cálculos particulares.

Palabras clave: Lógicas subestructurales; Teorías de la verdad; Reglas estructurales; Reglas operacionales.

\begin{abstract}
In past years, several substructural solutions to semantical paradoxes have been developed. In particular, nontransitive, noncontractive, nonmonotonic and nonreflexive theories have been proposed. However, when such solutions are presented using sequent-calculi it emerges what I call the problem of the dependence. In a nutshell, this problem consists in that it's not easy (or sometimes even possible) to distinguish between the structural rules and the other rules of the calculus. In this article, I will present in detail this problem and I show that even worst there
\end{abstract}


is a calculus such that it doesn't contain any structural rule but cannot nontrivially handle semantical vocabulary. Finally, I will delimit this problem and I will conclude that the correct methodology for preferring one specific substructural theory should be based on philosophical arguments (or in some case, on empirical bases), but not in the comparison between derivations in particular calculi.

Key words: Substructural Logics; Theories of Truth; Structural Rules; Operational Rules.

\section{Introducción}

El concepto de verdad ha sido estudiado de manera profunda a lo largo de la historia de la filosofía. Sin embargo, no fue hasta el siglo pasado cuando en 1933 Tarski intentó por primera vez utilizar métodos formales a los fines de construir lo que conocemos como una "teoría de la verdad" (Tarski, 1983). También fue Tarski quien notó que cualquier teoría formulada en lógica clásica y lo suficientemente expresiva para representar su propia sintaxis (una teoría aritmética débil) es incapaz de representar mediante un predicado su propio concepto de verdad. La razón de ello es que, para hacerlo, dicho predicado debería satisfacer algunos principios tales como el Esquema- $\mathrm{T}^{1}$, lo que lleva a paradojas.

La solución que muchos filósofos y lógicos han encontrado para solucionar este tipo de problemas ha consistido en formular teorías de la verdad utilizando como lógica de base una lógica no clásica y, de esta manera, incorporar las paradojas dentro de las teorías sin que estas últimas sean triviales. En los últimos años, el foco de estudio se ha posado sobre las teorías de la verdad basadas en lógicas subestructurales. Estas lógicas se caracterizan por el rechazo o restricción de alguna regla estructural, es decir una de aquellas reglas que estipulan las propiedades de la noción misma de consecuencia lógica. Las propuestas más comunes basadas en presentaciones en cálculos de secuentes consisten en el abandono de la regla de corte y de contracción, mientras que más recientemente también ha sido propuesto el abandono de reflexividad y de monotonía.

Algunos teóricos han ido un paso más allá en este sentido y han afirmado que de hecho alguna de estas reglas estructurales es $l a$ culpable de las paradojas. No solo eso, las derivaciones de las paradojas han sido utilizadas como evidencia de su culpabilidad. En

\footnotetext{
${ }^{1}$ Hay otras reglas equivalentes clásicamente que también se han propuesto en lugar del Esquema-T, tales como T-In y T-Out o las que veremos en las secciones que siguen.
} 
este artículo, siguiendo algunas consideraciones vertidas por Hjortland (2017), discutiremos esta metodología acentuando el hecho de que no es tan simple distinguir entre reglas que están siendo aplicadas de manera explícita en una derivación de aquellas que lo están haciendo implícitamente. Llamaré a este el problema de la dependencia de las soluciones subestructurales del marco en el que se presentan. Luego, mostraré en qué sentido este es un problema para las soluciones subestructurales y en qué sentido no lo es.

A los fines de llegar a ese punto, el artículo se estructura de la siguiente manera. En la Sección 2 presentaré un cálculo de secuentes para la lógica clásica y mostraré cómo las paradojas pueden ser derivadas formalmente. En la Sección 3, introduciré las principales soluciones subestructurales a las paradojas semánticas. En la Sección 4, en el mismo sentido que Hjortland (2017), desarrollaré el problema de la dependencia, esto es cuán dependiente son las soluciones subestructurales de las características generales de los cálculos en los que se presentan, en particular de la formulación de las reglas operacionales. A los fines de enfatizar esta dependencia, diseñaré un cálculo de secuentes que no exhibe de manera explícita ninguna regla estructural y sin embargo es trivial una vez que se le agrega el predicado veritativo. Luego, evaluaré si esta dependencia es un problema para dichas soluciones y desplegaré algunas líneas de respuesta. Finalmente, en la Sección 5 finalizaré con algunas conclusiones.

\section{Lógica clásica y paradojas}

Como hemos mencionado, del trabajo de Tarski se desprende que una teoría de la verdad clásica debe restringir las reglas de predicado veritativo. Lo que haremos ahora es presentar formalmente las paradojas. A lo largo de este artículo evitaremos la apelación a la semántica y nos restringiremos a las presentaciones en teorías de la prueba (en particular, en cálculos de secuentes), dado que las teorías subestructurales son en su mayoría presentadas de esta manera. ${ }^{2}$ Un cálculo de secuentes (Avron, 2003) sobre un lenguaje L es un sistema axiomático formulado sobre secuentes, no sobre fórmulas. Un secuente, por su lado, es un objeto de la forma $\Gamma \Rightarrow \Delta$, donde $\Gamma, \Delta$ son multiconjuntos ${ }^{3}$

\footnotetext{
${ }^{2}$ Por supuesto esta afirmación tiene excepciones tales como el de la lógica ST o TS que también son presentadas semánticamente (véase Cobreros et al., 2013) o la teoría no contractiva NC (Rosenblatt, 2019).

${ }^{3}$ Intuitivamente, un multiconjunto difiere de un conjunto en que el número de ocurrencias de un determinado elemento es relevante. Así, por ejemplo, los
} 
de fórmulas ${ }^{4} \mathrm{y} \Rightarrow$ es un símbolo que no pertenece al lenguaje objeto.

Definición 1. Sean $\Gamma, \Delta$ multiconjuntos finitos de fórmulas y sean $\varphi, \psi$ dos fórmulas. El cálculo $\mathrm{S}_{\mathrm{CL}}$ es definido por las siguientes reglas

\section{Reglas estructurales}

$$
\begin{array}{r}
\operatorname{Id} \frac{\phi^{A t} \Rightarrow \phi^{A t}}{} \\
\operatorname{KR} \frac{\Gamma \Rightarrow \Delta}{\Gamma \Rightarrow \Pi, \Delta} \\
\text { WR } \frac{\Gamma \Rightarrow \phi, \phi, \Delta}{\Gamma \Rightarrow \phi, \Delta} \\
\operatorname{Corte}^{A} \frac{\Gamma \Rightarrow \phi, \Delta \quad \Gamma, \phi \Rightarrow \Delta}{\Gamma \Rightarrow \Delta}
\end{array}
$$$$
\mathrm{KL} \frac{\Gamma \Rightarrow \Delta}{\Gamma, \Sigma \Rightarrow \Delta}
$$$$
\mathrm{WL} \frac{\Gamma, \phi, \phi \Rightarrow \Delta}{\Gamma, \phi \Rightarrow \Delta}
$$

Reglas operacionales

$$
\begin{aligned}
& \mathrm{L} \neg \frac{\Gamma \Rightarrow \phi, \Delta}{\neg \phi, \Gamma \Rightarrow \Delta} \\
& \mathrm{L} \wedge \frac{\Gamma, \phi \Rightarrow \Delta}{\Gamma, \phi \wedge \psi \Rightarrow \Delta} \\
& \mathrm{L} \wedge \frac{\Gamma, \psi \Rightarrow \Delta}{\Gamma, \phi \wedge \psi \Rightarrow \Delta} \\
& \mathrm{L} \vee \frac{\Gamma, \phi \Rightarrow \Delta \quad \Gamma, \psi \Rightarrow \Delta}{\Gamma, \phi \vee \psi \Rightarrow \Delta}
\end{aligned}
$$$$
\mathrm{R} \neg \frac{\Gamma, \phi \Rightarrow \Delta}{\Gamma \Rightarrow \neg \phi, \Delta}
$$$$
\mathrm{R} \wedge \frac{\Gamma \Rightarrow \phi, \Delta \quad \Gamma \Rightarrow \psi, \Delta}{\Gamma \Rightarrow \phi \wedge \psi, \Delta}
$$$$
\mathrm{R} \vee \frac{\Gamma \Rightarrow \phi, \Delta}{\Gamma \Rightarrow \phi \vee \psi, \Delta}
$$$$
\mathrm{R} \vee \frac{\Gamma \Rightarrow \psi, \Delta}{\Gamma \Rightarrow \phi \vee \psi, \Delta}
$$

donde $\varphi^{A t}$ es una oración atómica. Como es usual, el cálculo $\mathrm{S}_{\mathrm{CL}}$ es correcto y completo para la lógica clásica. Un condicional es definible a partir de las reglas de la negación y de la disyunción como es usual:

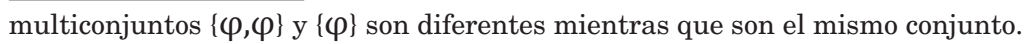

${ }^{4}$ Los secuentes también pueden ser presentados como pares de secuencias o de conjuntos de fórmulas. Aquí, utilizamos multiconjuntos dado que deseamos que intercambio esté absorbido en el sistema, pero no contracción. 


$$
\begin{array}{ll}
\mathrm{R} \rightarrow \frac{\Gamma, \phi \Rightarrow \Delta}{\Gamma \Rightarrow \phi \rightarrow \psi, \Delta} & \mathrm{R} \rightarrow \frac{\Gamma \Rightarrow \psi, \Delta}{\Gamma \Rightarrow \phi \rightarrow \psi, \Delta} \\
\mathrm{L} \rightarrow \frac{\Gamma \Rightarrow \phi, \Delta \quad \Gamma, \psi \Rightarrow \Delta}{\Gamma, \phi \rightarrow \psi \Rightarrow \Delta} &
\end{array}
$$

Lo que haremos ahora es construir una teoría de la verdad extendiendo este cálculo. En particular dado que estamos interesados en teorías de la verdad necesitamos conseguir autorreferencia. Para lograrlo, evitaremos apelar a aritmética, dado que eso haría más complejo el trabajo de lo que necesitamos para mostrar el punto de la dependencia de las lógicas subestructurales en sus presentaciones en cálculos de secuentes. Entonces, extenderemos el lenguaje proposicional L con un predicado $\operatorname{Tr}$ y un conjunto denumerable de constantes. Este nuevo lenguaje será llamado $\mathrm{L}^{+5}$. Cada constante que hemos agregado al lenguaje referirá a una y solo una fórmula del lenguaje $\mathrm{L}^{+} \mathrm{y}$ mediante el método de función auxiliar desarrollado en Beall, Glanzberg y Ripley (2018) y en Ripley (2014), tomaremos dichas funciones que generan oraciones paradójicas. En lo que sigue, $\lceil\varphi\rceil$ será usada como el nombre de $\varphi$, y $\operatorname{Tr}(\lceil\varphi\rceil)$ significará que la oración $\varphi$ es verdadera. ${ }^{6}$

Una vez que hemos enriquecido el lenguaje L y construido el lenguaje $\mathrm{L}^{+}$, nos gustaría que el predicado de verdad agregado satisficiera ciertas reglas que capturen la noción ingenua de verdad, es decir su comportamiento en el lenguaje natural. Siguiendo la tradición, (e.g. Beall, 2009 y Beall, Glanzberg, \& Ripley, 2018) tomaremos la noción de transparencia como la propiedad que un predicado de verdad apropiado debe satisfacer. En términos de reglas, la idea es que se garantice la intersustitutividad de una oración por su predicación de verdad en todo contexto no opaco, es decir de la oración $\varphi$ por la oración $\operatorname{Tr}([\varphi])$. Así, recordando los ejemplos tradicionales, la transparencia nos permite reemplazar la oración The snow is white por la oración 'The snow is white' is true, siempre que estemos evaluando su contenido semántico en contextos no problemáticos. Formalmente,

\footnotetext{
${ }^{5}$ Por supuesto, aquí suponemos las definiciones usuales de fórmula bien formada, fórmulas atómicas, fórmulas complejas, etc.

${ }^{6}$ Hablando estrictamente, $\lceil\varphi\rceil$ no está en el lenguaje, sino que existe una constante que refiere a $\varphi$ mediante la función auxiliar. Aquí, dado que estamos usando las constantes únicamente para poder hablar de las oraciones, omitiremos esta distinción y usaremos el nombre de las oraciones en lugar de las constantes.
} 
Definición 2. Sea $\mathrm{S}_{\mathrm{CLTT}}$ el cálculo resultante de agregar a $\mathrm{S}_{\mathrm{CL}}$ sobre $\mathrm{L}^{+}$, las siguientes dos reglas ${ }^{7}$ :

$$
\operatorname{LTr} \frac{\phi, \Gamma \Rightarrow \Delta}{\operatorname{Tr}(\ulcorner\phi\urcorner), \Gamma \Rightarrow \Delta}
$$

$$
\mathrm{R} \operatorname{Tr} \frac{\Gamma \Rightarrow \phi, \Delta}{\Gamma \Rightarrow \operatorname{Tr}(\ulcorner\phi\urcorner), \Delta}
$$

Definimos la teoría CLTT como el conjunto de secuentes derivables a partir del cálculo $\mathrm{S}_{\mathrm{CuTT}}$. Así, tenemos una teoría clásica de la verdad aparentemente muy débil ${ }^{8}$. Sin embargo es lo suficientemente fuerte como para derivar cualquier secuente. Es decir, es trivial.

\section{Observación 3. CLTT es trivial.}

Prueba. Una vez que admitimos que ciertas oraciones paradójicas pueden ser expresadas en la teoría es posible derivar una contradicción. Por ejemplo, tomemos la oración del mentiroso, i.e. una oración que dice de sí misma que no es verdadera: $\lambda$ equivalente a $\neg \operatorname{Tr}([\lambda])$. Si tenemos esta oración en el lenguaje entonces podemos construir la siguiente derivación en $\mathrm{S}_{\text {CLTT }}{ }^{9}$ :

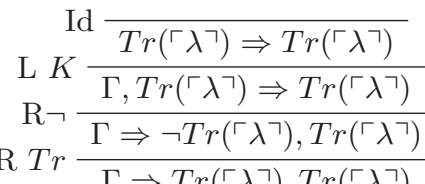

$$
\begin{aligned}
& \mathrm{R} W \frac{\Gamma \Rightarrow \operatorname{Tr}(\ulcorner\lambda\urcorner), \operatorname{Tr}(\ulcorner\lambda\urcorner)}{\Gamma \Rightarrow \operatorname{Tr}(\ulcorner\lambda\urcorner)} \\
& \mathrm{R} K \frac{\Gamma \Rightarrow \operatorname{Tr}(\ulcorner\lambda\urcorner)}{\Gamma \Rightarrow \operatorname{Tr}(\ulcorner\lambda\urcorner), \Delta}
\end{aligned}
$$

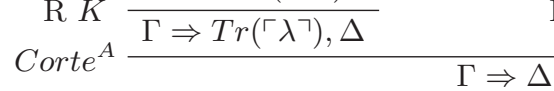

con $\Gamma$ y $\Delta$, dos multiconjuntos cualquiera de fórmulas.

Esta es una derivación de la famosa paradoja del mentiroso. De dicha derivación se puede observar que además de las reglas para la negación y para el predicado veritativo, todas las reglas estructurales han sido aplicadas.

${ }^{7}$ La doble línea en las reglas significa que estas pueden ser leídas desde arriba hacia abajo, pero también desde abajo hacia arriba.

${ }^{8}$ Aquí, nos estamos refiriendo a la fortaleza expresiva. En otras palabras, no estamos asumiendo una teoría aritmética.

${ }^{9}$ Aun cuando es común presentar esta paradoja, desde un punto de vista de teoría de la prueba existen diferentes presentaciones en la literatura. Aquí tomamos solo una de ellas, sobre la que volveremos en las secciones siguientes. 
Hay otra célebre paradoja semántica que ya no incluye el uso de la negación sino del condicional ${ }^{10}$ : la paradoja de Curry. Supongamos que hay una oración $\kappa$, equivalente a $\operatorname{Tr}(\lceil\kappa\rceil) \rightarrow \gamma$, donde $\gamma$ es una oración arbitraria del lenguaje. ${ }^{11}$ Entonces, podemos construir una prueba de trivialidad como sigue:

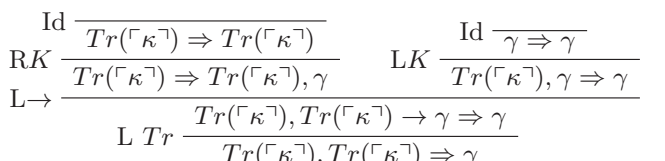

$$
\begin{aligned}
& \text { L } W \frac{\operatorname{Tr}(\ulcorner\kappa\urcorner), \operatorname{Tr}(\ulcorner\kappa\urcorner) \Rightarrow \gamma}{\operatorname{Tr}(\ulcorner\kappa\urcorner) \Rightarrow \gamma} \\
& \text { L } K \frac{\Rightarrow \gamma}{\sum \Rightarrow \gamma}
\end{aligned}
$$

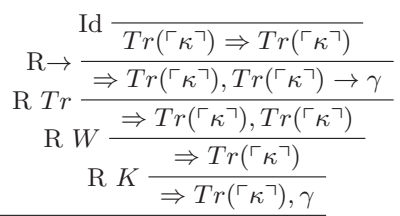

con $\Sigma$, un multiconjunto arbitrario de fórmulas y $\Gamma$ algún multiconjunto de fórmulas que contenga a $\gamma$. Como en el caso de la derivación de la paradoja del mentiroso, aquí también todas las reglas estructurales han sido aplicadas, junto con las del condicional y las del predicado veritativo.

Así, muchos autores a los fines de evitar estas derivaciones han optado por no modificar las reglas del predicado veritativo, sino restringir o abandonar alguna de las reglas estructurales del cálculo $\mathrm{S}_{\mathrm{CL}}$ (o cálculos equivalentes) y así adoptar una sublógica de la lógica clásica. ${ }^{12}$ Estas son las llamadas teorías subestructurales de la verdad. En la sección siguiente, mencionaremos y describiremos someramente algunas de ellas.

\section{Las soluciones subestructurales}

Las soluciones subestructurales a las paradojas semánticas consisten en el abandono de alguna (o algunas) de las reglas estructurales. En este sentido, como hemos visto hay cuatro reglas estructurales: reflexividad, corte, contracción y monotonía.

${ }^{10}$ Siendo estrictos, aquí estamos tomando el condicional como definido a partir de la negación y de la disyunción. Sin embargo, esto es incidental en esta presentación y la paradoja de Curry puede incluso ser formulada en un lenguaje sin negación.

${ }^{11}$ Tomamos $\gamma$ como una oración del lenguaje que no desearíamos probar. Más formalmente, podríamos construir una oración de Curry para cada oración del lenguaje y, así, podríamos derivar todas las oraciones del lenguaje.

12 Esta aseveración es verdadera excepto para ST, dado que esta lógica es equivalente a la lógica clásica en el conjunto de inferencias válidas, pero no en el conjunto de metainferencias. Véase e.g. Cobreros et al. (2012). 
Entre quienes proponen el abandono de la regla de contracción, se destacan: Mares y Paoli (2014), Shapiro (2011), Brady (2006), Murzi y Shapiro (2015), Weber (2014), Caret y Weber (2015), Zardini (2011, 2013, 2014), y más recientemente, Rosenblatt (2019). En estos enfoques, usualmente las siguientes derivaciones $\lambda, \lambda \Rightarrow y \Rightarrow \lambda, \lambda$ están permitidas, pero dado que contracción está ausente, no es posible derivar de dichos secuentes $\lambda \Rightarrow \mathrm{ni} \Rightarrow \lambda$.

Por otro lado, se encuentran aquellos teóricos que propusieron el abandono de la regla de corte, entre quienes podemos mencionar a Weir (2013), Tennant (2015), Cobreros et al. (2012, 2013) y Ripley (2012). En particular Cobreros et al. y Ripley proponen la adopción de la lógica ST y consideran que la regla de corte no es correcta en contextos en los que hay vocabulario semántico (y también vocabulario vago). Así, por ejemplo, en algunas circunstancias los autores mencionados aceptan la derivación de dos secuentes tales como $\varphi \Rightarrow \psi$ y $\psi \Rightarrow \gamma$, pero rechazan que de ellos se pueda derivar el secuente $\varphi \Rightarrow \gamma \cdot{ }^{13}$ Un hecho interesante respecto de las teorías basadas en la lógica ST es que es posible derivar todos los secuentes clásicos, esto es ST coincide inferencialmente con la lógica clásica. Esto se debe a que la lógica ST es presentada desde un punto de vista de teoría de la prueba como el cálculo resultante de eliminar la regla de corte del cálculo LK para la lógica clásica, presentado por Gentzen. Como resultado del célebre teorema de eliminación de corte, el famoso Hauptsatz, todo secuente derivable en LK puede ser probado sin utilizar la regla de corte. Sin embargo, una vez que se agrega un predicado de verdad, ambos sistemas obviamente difieren (véase Ripley, 2012 o Cobreros et al., 2013).

En tercer lugar, French (2016), Fjellstad (2015), Nicolai y Rossi (2018) y Schroeder-Heister (2012, 2016), entre otros, han propuesto el abandono de la regla de reflexividad, el único axioma del cálculo de secuentes. En particular, French propone la adopción de la lógica TS. En el cálculo para TS, obviamente al no haber axiomas ningún secuente es derivable. En particular, $\lambda \Rightarrow \lambda$ no es derivable.

Finalmente, más recientemente Da Ré (2020) ha propuesto la adopción de una lógica no monotónica a los fines de lidiar con vocabulario semántico. En particular, en dicha lógica las demás reglas estructurales son aceptadas y, por lo tanto, una vez que agregamos oraciones paradójicas tenemos una derivación del secuente vacío. Sin

${ }^{13}$ Como veremos más adelante, dependiendo del caso, la regla de corte puede ser formulada con contextos compartidos (la regla $\operatorname{Corte}^{\mathrm{A}}$ ) o con contextos independientes. Esta regla será llamada Corte ${ }^{\mathrm{M}}$. 
embargo, dado que las reglas de monotonía no son admisibles no es posible construir una derivación a partir de dicho secuente a los fines de trivializar la teoría.

Habiendo esquemáticamente esbozado (o en algunos casos simplemente mencionado) las principales soluciones subestructurales a las paradojas, estamos en posición de presentar el problema de la dependencia de dichos enfoques. Antes de ir hacia eso, es importante realizar la siguiente aclaración: el problema que aquí presentaremos solo se aplica a aquellas soluciones que son planteadas utilizando cálculos de secuentes, quedando exentas todas aquellas propuestas que son presentadas semánticamente. ${ }^{14}$

\section{La dependencia de las soluciones subestructurales}

Como hemos mencionado, las soluciones subestructurales a las paradojas surgen como rivales a las soluciones estructurales, esto es las soluciones que no modifican ninguna regla estructural, sino únicamente reglas operacionales. Usualmente, las soluciones subestructurales son presentadas mediante un cálculo de secuentes de forma tal que, en dichos sistemas, alguna regla estructural es restringida o abandonada. En otras palabras, aquellos que adoptan soluciones subestructurales, en general, argumentan que sus soluciones modifican alguna regla estructural, pero conservando las reglas operacionales. Sin embargo, en el mismo sentido que lo ha marcado Hjortland $(2017)^{15}$, considero que no se ha hecho suficiente hincapié en el siguiente punto: toda solución subestructural impone restricciones sobre las reglas operacionales. En otras palabras, no existen las soluciones subestructurales puras. No slo eso, además, las soluciones subestructurales están esencialmente relativizadas al cálculo de secuentes en el que se presentan. Esto es lo que llamaremos el problema de la dependencia de las soluciones subestructurales. A continuación, ahondaremos en este problema. Luego, delimitaremos dicho problema

${ }^{14}$ En particular, las teorías de la verdad ST y TS cuando son presentadas como relaciones de consecuencia mixta sobre esquemas trivaluados.

${ }^{15}$ En esta sección algunos de los resultados que presento también se encuentran en Hjortland (2017). Sin embargo, cabe mencionar algunas diferencias. En primer lugar, el autor no considera la regla $\operatorname{Corte}^{A}$, ni el cálculo que hemos presentado en la Sección 2, ni la solución no monotónica a las paradojas. Esto limita las conclusiones que extrae a la formulación de las reglas operacionales. Por otro lado, el autor compara las soluciones operacionales con las soluciones subestructurales. Sin embargo, aquí no intentamos realizar ninguna comparación de ese tipo, sino que nos enfocamos en las soluciones subestructurales y mostramos el alcance de los problemas que aquí se presentan respecto de estas soluciones. 
y discutiremos en qué sentido puede ser disuelto. Comencemos, entonces con una cita que encontramos en la bibliografía, pero creemos representativa. Dutilh Novaes y French (2018, p.143) argumentan: "Left Weakening... is not discussed in the recent literature on substructural approaches to paradox, as it does not play a prominent role in typical derivations of the Liar, Curry etc.”. Dejando de lado el contenido de dicha afirmación, lo que nos gustaría enfatizar es el hecho de que los autores toman la idea de typical derivations (derivaciones típicas) como evidencia de la presencia o ausencia de alguna regla estructural en algún razonamiento paradójico. Este criterio metodológico omite el hecho de que una regla estructural (o alguna instancia de ella) puede ser absorbida por reglas operacionales.

A los fines de ilustrar este punto, recordemos la distinción entre reglas aditivas y reglas multiplicativas. Una vez que monotonía y/o contracción no son admisibles en un cálculo de secuentes, podemos formular de diversas maneras las reglas operacionales para los conectivos. Las dos formas más usuales son mediante reglas aditivas y mediante reglas multiplicativas. Comencemos con las dos reglas para $\otimes$ :

$$
\mathrm{R} \otimes \frac{\Gamma \Rightarrow \phi, \Delta \quad \Sigma \Rightarrow \Pi, \psi}{\Gamma, \Sigma \Rightarrow \psi \otimes \phi, \Pi, \Delta} \quad \mathrm{L} \otimes \frac{\phi, \psi, \Gamma \Rightarrow \Delta}{\phi \otimes \psi, \Gamma \Rightarrow \Delta}
$$

donde $\otimes$ es el símbolo para la conjunción multiplicativa. Por otro lado, podríamos tomar las reglas (aditivas) que hemos presentado para la conjunción $(\Lambda)$ en el cálculo $\mathrm{S}_{\mathrm{CL}}$ (véase Sección 2):

$$
\mathrm{L} \wedge \frac{\phi, \Gamma \Rightarrow \Delta}{\phi \wedge \psi, \Gamma \Rightarrow \Delta} \quad \mathrm{L} \wedge \frac{\psi, \Gamma \Rightarrow \Delta}{\phi \wedge \psi, \Gamma \Rightarrow \Delta}
$$

y la regla a la derecha:

$$
\mathrm{R} \wedge \frac{\Gamma \Rightarrow \phi, \Delta \quad \Gamma \Rightarrow \Delta, \psi}{\Gamma \Rightarrow \psi \wedge \phi, \Delta}
$$

Así, tanto $\wedge$ como $\otimes$ son solo dos símbolos diferentes para representar la conjunción. En lógica clásica, ambos pares de reglas son interderivables y por lo tanto no es relevante cuál de ellos elijamos a la hora de formular un cálculo. Esto es así dado que una vez que monotonía y contracción están presentes tenemos las siguientes derivaciones (asumiendo las reglas de la izquierda correspondientes): 


$$
\begin{gathered}
\mathrm{L}-\mathrm{R} K \frac{\Gamma \Rightarrow \phi, \Delta}{\mathrm{R} \wedge \frac{\Sigma, \Sigma \Rightarrow \phi, \Delta, \Pi}{\Gamma, \Sigma \Rightarrow \psi \wedge \phi, \Pi, \Delta} \quad \operatorname{L-R} K \frac{\Sigma \Rightarrow \Pi, \psi}{\Gamma, \Sigma \Rightarrow \psi, \Delta, \Pi}} \\
\mathrm{R} \otimes \frac{\Gamma \Rightarrow \phi, \Delta \quad \Gamma \Rightarrow \Delta, \psi}{\mathrm{L}-\mathrm{R} W \frac{\Gamma, \Gamma \Rightarrow \psi \otimes \phi, \Delta, \Delta}{\Gamma \Rightarrow \psi \otimes \phi, \Delta}}
\end{gathered}
$$

Lo mismo podríamos probar para las reglas aditivas $\mathrm{y}$ multiplicativas de introducción de la conjunción a la izquierda.

Sin embargo, una vez que monotonía y/o contracción están ausentes la manera en la que formulamos las reglas deviene relevante, dado que ello implicará cuánto de las reglas estructurales mencionadas estaremos absorbiendo. Por ejemplo, la regla a la derecha correspondiente a $\wedge$ absorbe contracción, mientras que $\otimes$ absorbe monotonía.

No solo eso, podríamos inclusive presionar más el punto al tomar una formulación mixta de las reglas de los conectivos. Por ejemplo, consideremos las siguientes reglas (aditivas) a izquierda:

$$
\mathrm{L} \sqcap \frac{\phi, \Gamma \Rightarrow \Delta}{\phi \sqcap \psi, \Gamma \Rightarrow \Delta} \quad \mathrm{L} \sqcap \frac{\psi, \Gamma \Rightarrow \Delta}{\phi \sqcap \psi, \Gamma \Rightarrow \Delta}
$$

y la regla (multiplicativa) a la derecha:

$$
\mathrm{R} \sqcap \frac{\Gamma \Rightarrow \phi, \Delta \quad \Sigma \Rightarrow \Pi, \psi}{\Gamma, \Sigma \Rightarrow \psi \sqcap \phi, \Delta, \Pi}
$$

Con Corte $^{M 16}$ y reflexividad, estas reglas son suficientes para recuperar monotonía a la izquierda como regla derivable:

$$
\begin{aligned}
& \begin{aligned}
&\left(\operatorname{con} \Delta=\Delta^{\prime}-\{\phi\}\right) \frac{\Gamma \Rightarrow \Delta^{\prime}}{\Gamma \Rightarrow \Delta, \phi} \quad \psi \Rightarrow \psi \\
& \operatorname{R} \sqcap \frac{}{\Gamma \Rightarrow \Delta} \quad \mathrm{L} \sqcap \frac{\phi \Rightarrow \phi}{\phi \sqcap \psi \Rightarrow \phi} \\
& \text { Corte }^{M} \frac{\Gamma, \psi \Rightarrow \Delta, \phi \sqcap \psi}{\Gamma, \psi \Rightarrow \phi, \Delta}
\end{aligned} \\
& \left(\operatorname{con} \Delta=\Delta^{\prime}-\{\phi\}\right) \frac{\Gamma, \psi \Rightarrow \phi, \Delta}{\Gamma, \psi \Rightarrow \Delta^{\prime}}
\end{aligned}
$$

Cabe destacar que en la anterior derivación, asumimos que $\Delta$ es no vacío, por lo que en rigor probamos algo más débil que la derivabilidad de monotonía a izquierda. En el caso que $\Delta$ fuera vacío, si no tuviéramos

${ }^{16}$ Corte $^{M}$ es la regla de corte formulada con contextos independientes. 
más reglas que las reglas de la conjunción presentadas, monotonía a izquierda sería admisible, aunque no derivable. ${ }^{17}$ Consideraciones simétricas (apelando a reglas duales) pueden realizarse para recuperar la regla de monotonía a derecha.

Por otro lado, si formulamos las reglas mixtas para la conjunción de una manera dual, recuperamos la regla a la izquierda de contracción. Consideremos las siguientes reglas:

$$
\mathrm{L} \curlywedge \frac{\phi, \psi, \Gamma \Rightarrow \Delta}{\phi \curlywedge \psi, \Gamma \Rightarrow \Delta} \quad \mathrm{R} \curlywedge \frac{\Gamma \Rightarrow \psi, \Delta \quad \Gamma \Rightarrow \phi, \Delta}{\Gamma \Rightarrow \phi \curlywedge \psi, \Delta}
$$

donde la regla a la izquierda es multiplicativa, mientras que la regla de la derecha es aditiva. Con $\operatorname{Corte}^{M}$, podemos derivar la regla izquierda de contracción ${ }^{18}$ :

$$
\mathrm{R} \curlywedge \frac{\phi \Rightarrow \phi \quad \phi \Rightarrow \phi}{\text { Corte }^{M} \frac{\mathrm{\phi \Rightarrow \phi \curlywedge \phi}}{\Gamma, \phi \Rightarrow \Delta}}
$$

Respecto de la regla derecha, una derivación similar puede ser presentada.

Así, lo que estos ejemplos muestran es que no es inocente la manera en que las reglas operacionales son formuladas en cálculos que pretenden ser utilizados para desarrollar teorías subestructurales. Hasta aquí, podría pensarse que estas relaciones son abstractas. Sin embargo, a continuación lo ilustraremos con una derivación. Tomemos la derivación de la paradoja del mentiroso presentada en la Sección 2. Luego de la derivación, hemos afirmado que todas las reglas estructurales son aplicadas en dicha derivación. Sin embargo, consideremos la siguiente derivación alternativa de la paradoja en $\mathrm{S}_{\mathrm{CLTT}}$, donde contracción no es aplicada explícitamente. Primero, derivamos $\Rightarrow \operatorname{Tr}([\lambda])$ :

$$
\begin{aligned}
& \mathrm{Id} \frac{\operatorname{Tr}(\ulcorner\lambda\urcorner) \Rightarrow \operatorname{Tr}(\ulcorner\lambda\urcorner)}{\mathrm{R}} \\
& \mathrm{R} \operatorname{Tr} \frac{}{\Rightarrow \operatorname{Tr}(\ulcorner\lambda\urcorner), \neg \operatorname{Tr}(\ulcorner\lambda\urcorner)} \operatorname{Id} \frac{\operatorname{Tr}(\ulcorner\lambda\urcorner) \Rightarrow \operatorname{Tr}(\ulcorner\lambda\urcorner)}{\Rightarrow \operatorname{Tr}(\ulcorner\lambda\urcorner), \operatorname{Tr}(\ulcorner\lambda\urcorner)} \\
& \text { Corte }^{A} \frac{\operatorname{Tr}(\ulcorner\lambda\urcorner)}{\Rightarrow \operatorname{Tr}(\ulcorner)}
\end{aligned}
$$

${ }_{17}$ Este hecho puede probarse por inducción sobre el alto de las derivaciones. Dejamos dicha prueba para el lector.

${ }_{18}^{18}$ Una lógica con las reglas operacionales formuladas de esta manera y con la regla de corte admisible es usada por Rosenblatt (2019) para lidiar con paradojas. 
Luego, probamos $\operatorname{Tr}([\lambda]) \Rightarrow$ :

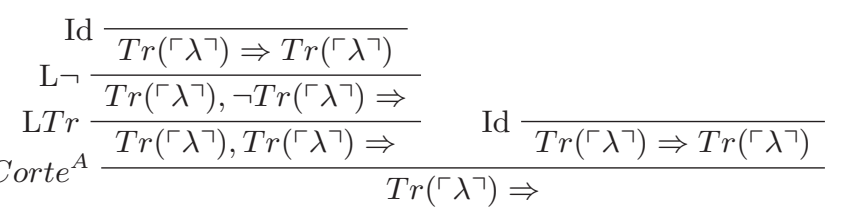

Finalmente, aplicamos la regla de $\operatorname{Corte}^{A}$ y monotonía y podemos derivar cualquier secuente.

Así, tenemos una derivación de la paradoja del mentiroso que no hace uso de las reglas de contracción. Si consideráramos la cita de Dutilh Novaes y French que hemos presentado al comienzo de esta sección y asumiéramos que las derivaciones típicas de las paradojas en cálculos de secuentes fueran del tipo que he presentado anteriormente, entonces no habría evidencia en favor de que contracción está presente en los razonamientos paradójicos, lo cual contradice la tradición de teóricos no contractivos que hemos mencionado.

Por lo tanto, creo que el hecho de que una regla estructural como contracción participe de manera esencial de un razonamiento paradójico no debería depender de una derivación particular de dicha paradoja en un cálculo específico. En este sentido, aun cuando contracción no esté siendo aplicada de manera explícita en la derivación, un teórico no contractivista podría argumentar que de hecho contracción está siendo aplicada, solo que de manera implícita al utilizar reglas aditivas para las conectivas y la regla de corte con contextos compartidos. En otras palabras, no es evidencia para determinar que una regla estructural no está presente en un razonamiento paradójico el hecho de presentar una derivación de dicha paradoja. En este caso, las reglas aditivas y de contexto compartido absorben suficiente contracción como para que no sea necesario aplicar explícitamente dicha regla en la derivación de la paradoja. Esto pareciera ser particularmente problemático para las reglas de contracción y de monotonía. Sin embargo, estas consideraciones pueden ser extendidas a las teorías no transitivas y no reflexivas. Los teóricos no reflexivos usualmente rechazan el agregado de axiomas ${ }^{19}$ mientras que los teóricos no transitivos tienden a rechazar reglas de eliminación ${ }^{20}$.

${ }^{19}$ Por ejemplo, French (2016) rechaza la regla VD en el contexto de teorías naive de la validez, dado que siendo un axioma, absorbe reflexividad.

${ }^{20}$ De hecho, Ripley (2015a) argumenta contra algunas reglas, como las de Supertonk, dado que son reglas de eliminación (y por lo tanto absorben corte). 
En pocas palabras, lo que he mostrado hasta aquí es que las soluciones subestructurales a las paradojas, cuando son presentadas mediante un cálculo de secuentes, son sensibles a la manera en la que son formuladas las reglas para los conectivos.

A continuación, ilustraré aún más este punto presentando un cálculo en el cual ninguna regla estructural está explícitamente formulada y que, sin embargo, una vez intentamos construir una teoría de la verdad deviene trivial. Comencemos con una lógica $\mathbf{M}$ definida como el conjunto de secuentes derivables por el siguiente cálculo.

Definición 4. Sean $\Gamma, \Delta$, П, y $\Sigma$ multiconjuntos finitos de fórmulas y sean $\varphi$ y $\psi$ dos fórmulas de $\mathrm{L}$. El cálculo $\mathrm{S}_{\mathrm{M}}$ consiste en las siguientes reglas:

$$
\begin{array}{cc}
\mathrm{R} \neg \frac{\Gamma, \phi \Rightarrow \Delta}{\Gamma \Rightarrow \Delta, \neg \phi} & \mathrm{L} \neg \frac{\Gamma \Rightarrow \Delta, \phi}{\neg \phi, \Gamma \Rightarrow \Delta} \\
\mathrm{R} \multimap \frac{\Gamma, \phi \Rightarrow \psi, \Delta}{\Gamma \Rightarrow \Delta, \phi \multimap \psi} & \mathrm{L} \multimap \frac{\Gamma \Rightarrow \phi, \Delta \quad \psi, \Sigma \Rightarrow \Pi}{\phi \multimap \psi, \Gamma, \Sigma \Rightarrow \Delta, \Pi} \\
m M P \frac{\Gamma \Rightarrow \phi \multimap \psi, \Delta \quad \Gamma \Rightarrow \phi, \Delta}{\Gamma \Rightarrow \psi, \Delta}
\end{array}
$$

En primer lugar, cabe resaltar que el cálculo así presentado no contiene de manera explícita ninguna regla estructural. De hecho, dado que reflexividad en particular no está presente el conjunto de secuentes derivables es vacío. En segundo lugar, la regla $m M P$ por meta Modus Ponens ${ }^{21}$ es una regla de eliminación para el condicional ${ }^{22}$.

Por lo tanto, si las reglas estructurales fueran las culpables de las paradojas, al estar ausentes en este cálculo, podríamos construir una teoría de la verdad no trivial sobre él. Sin embargo, agregar un predicado de verdad transparente y una oración paradójica con sus axiomas es suficiente para trivializar la teoría.

Definición 5. Sean $\Gamma, \Delta$, П, y $\Sigma$ multiconjuntos finitos de fórmulas

\footnotetext{
${ }^{21}$ Esta regla ha sido discutida en la bibliografía, especialmente en la disputa entre lógicos no contractivos y no transitivos. Lo que resulta interesante es que ninguno de ellos puede aceptar este tipo de reglas, dado que absorbe contracción y corte. Véase e.g. Zardini (2013), Fjellstad (2016) y Ripley (2015).

${ }^{22}$ Nótese que las reglas $(\mathrm{R}-0)$ y $(\mathrm{L} \rightarrow)$ son las correspondientes al condicional multiplicativo.
} 
y sean $\varphi$ y $\psi$ dos fórmulas de $L^{+}$. El cálculo $\mathrm{S}_{\mathrm{MTT}}$ consiste en agregar a $\mathrm{S}_{\mathbf{M}}$ las reglas LTr yRTr (véase Definición 2) y los siguientes axiomas:

$$
\text { Curry }-\operatorname{In} \frac{}{\operatorname{Tr}(\ulcorner\kappa\urcorner) \Rightarrow \operatorname{Tr}(\ulcorner\kappa\urcorner) \multimap \gamma} \quad \text { Curry }- \text { Out } \frac{}{\operatorname{Tr}(\ulcorner\kappa\urcorner) \multimap \gamma \Rightarrow \operatorname{Tr}(\ulcorner\kappa\urcorner)}
$$

donde $\kappa$ es la oración de Curry definida en la Sección 2, i.e. una oración que es equivalente a $\operatorname{Tr}(\mid \kappa\rceil) \rightarrow \gamma$, donde $\gamma$ es cualquier oración que no quisiéramos probar en la teoría. ${ }^{23}$

Así, las reglas del cálculo $\mathrm{S}_{\mathrm{MTT}}$ consisten en las reglas de $\mathrm{S}_{\mathrm{M}}$, más las reglas del predicado veritativo y los axiomas de la oración de Curry, representando la idea de que la teoría puede lidiar con oraciones paradójicas. Esto es suficiente para trivializar la teoría, como veremos a continuación. Sea $\mathrm{D}_{0}$ la siguiente derivación:

$$
\begin{array}{r}
\text { Curry }- \text { Out } \frac{}{\operatorname{Tr}(\ulcorner\kappa\urcorner) \multimap \gamma \Rightarrow \operatorname{Tr}(\ulcorner\kappa\urcorner)} \\
\mathrm{LTr} \frac{\operatorname{Tr}(\ulcorner\kappa\urcorner) \Rightarrow \operatorname{Tr}(\ulcorner\kappa\urcorner)}{\mathrm{R} \multimap \frac{\operatorname{Tr}(\ulcorner\kappa\urcorner) \Rightarrow \gamma}{\Rightarrow \operatorname{Tr}(\ulcorner\kappa\urcorner) \multimap \gamma}}
\end{array}
$$

De $\mathrm{D}_{0}$, podemos obtener $\mathrm{D}_{1}$ :

$$
\operatorname{RTr} \frac{\mathcal{D}_{0}}{\frac{\vdots}{\Rightarrow \operatorname{Tr}(\ulcorner\kappa\urcorner) \multimap \gamma}} \frac{}{\Rightarrow \operatorname{Tr}(\ulcorner\kappa\urcorner)}
$$

y juntando ambas derivaciones podemos derivar $\gamma$ :

${ }^{23}$ Es importante señalar que ni Curry-In ni Curry-Out son instancias del axioma de identidad. De hecho, utilizando el lenguaje formal mencionado en la nota al pie $\left.6, \operatorname{Tr}\left(\Gamma_{\mathrm{K}}\right\rceil\right) \multimap \gamma$ y $\kappa$ no son más que la misma oración, por lo que $\left.\left.\operatorname{Tr}\left(\Gamma_{\kappa}\right\rceil\right) \rightarrow \gamma \mathrm{y} \operatorname{Tr}\left(\Gamma_{\kappa}\right\rceil\right)$ son objetos sintácticos distintos. De hecho, Curry-In y Curry-Out son instancias de las reglas que usualmente se conocen como T-In y T-Out (las cuales son obviamente aceptables en un contexto de teorías naive). Agradezco a un revisor anónimo por indicarme este punto. 


$$
\begin{array}{cc}
\mathcal{D}_{0} & \mathcal{D}_{1} \\
\frac{\vdots}{\Rightarrow \operatorname{Tr}(\ulcorner\kappa\urcorner) \multimap \gamma} & \frac{\vdots}{\Rightarrow \operatorname{Tr}(\ulcorner\kappa\urcorner)} \\
\Rightarrow \gamma &
\end{array}
$$

dado que $\gamma$ es arbitraria, podríamos derivar cualquier oración del lenguaje $\mathrm{L}^{+}$. Por lo tanto la teoría resultante no es adecuada para lidiar con vocabulario semántico. ${ }^{24}$ Sin embargo, ninguna regla estructural ha sido utilizada en la derivación de manera explícita.

¿Es este un problema para las soluciones subestructurales? Veamos el siguiente razonamiento: si chequear que una regla estructural no es aplicada en una derivación fuera evidencia para argumentar que dicha regla no es la culpable de los razonamientos paradójicos, dado que hemos mostrado que hay derivaciones en las que ninguna regla estructural es aplicada, la conclusión sería que ninguna regla estructural es culpable de dichos fenómenos. ${ }^{25}$ Sin embargo, lo que creo que debe desprenderse de este análisis no es la conclusión del anterior razonamiento, sino la falsedad de una de sus premisas, esto es el carácter evidencial de la aplicación explícita de reglas en las derivaciones. En este sentido, creo que todas las reglas estructurales están presentes en todas las derivaciones de paradojas, solo que, dependiendo del sistema de prueba y la formulación de las reglas del cálculo, algunas lo están de manera explícita, mientras que otras se encuentran absorbidas en la formulación de las otras reglas del cálculo. En otras palabras, no hay razones lógicas para señalar a una regla estructural en particular como la culpable de una derivación de una paradoja semántica, sino que todas las reglas estructurales están en mayor o menor medida asumidas en la formulación de las reglas del cálculo en el que dicha derivación es llevada a cabo. ${ }^{26}$ Sin embargo, esto no implica que de hecho no haya

${ }^{24}$ Una derivación similar es presentada por Zardini (2013). La manera en la que evita el problema es rechazando la regla mMP argumentando que no representa la idea de Modus Ponens. Esta discusión es ajena al presente artículo.

${ }^{25}$ De hecho, hemos probado algo más fuerte: la existencia de una derivación en la que ninguna regla estructural ha sido aplicada. Sin embargo, el punto podría mostrarse simplemente presentando distintas derivaciones en cada una de las cuales no se aplica alguna de las reglas estructurales. Si este procedimiento fuera exhaustivo, la afirmación estaría probada. Agradezco a un revisor anónimo por haberme señalado esta posible alternativa.

${ }^{26}$ Obviamente, del cálculo M que he presentado y la teoría de la verdad resultante, no se sigue que toda derivación contenga implícitamente o explícitamente una aplicación de cada regla estructural. Lo que he mostrado meramente es compatible 
una (o más de una) regla que sea la culpable de los razonamientos paradójicos. Puesto en otros términos, el hecho de que las derivaciones particulares en cálculos de secuentes específicos no sean evidencia para mostrar la preponderancia de una solución subestructural por sobre otra no implica que no exista otro tipo de evidencia que permita dirimir esta disputa (sobre esto volveremos más abajo). En última instancia, podemos recordar la afirmación de Ripley (2015b, p. 310): "Rather than rushing from paradox to paradox making ad hoc modifications, these substructural approaches grapple with the paradoxes where they live: in the basic features of argumentation".

Este párrafo resume por qué las soluciones subestructurales han sido tan estudiadas y por qué son superaciones de las soluciones puramente operacionales: permiten dar una solución unificada a una gran variedad de paradojas, apuntando al núcleo mismo del razonamiento, esto es a la noción de consecuencia lógica. Sin embargo, como hemos mostrado, el hecho de que un determinado formalismo logre dar cuenta de un variopinto abanico de razonamientos clásicamente problemáticos, no es suficiente para explicar, en un sentido más sustantivo, cuál es el rol que dicha regla estructural juega en dichos razonamientos. Puesto en otros términos, aun cuando aceptáramos cuáles son las reglas formales que debe obedecer un predicado problemático, como el predicado veritativo, y además aceptáramos que en las derivaciones de las paradojas se aplica una determinada regla estructural, ello sin embargo no sería suficiente para afirmar que la culpable de dicha derivación o de dicho razonamiento problemático sea esa regla y no cualquier otra.

En esta línea de pensamiento, creo que todas las soluciones subestructurales están, desde un punto de vista formal, en igualdad de condiciones. En otros términos, dado que cualquier derivación paradójica puede ser bloqueada mediante el abandono de cualquier regla estructural, no existen razones puramente formales para afirmar la superioridad de una solución subestructural por sobre otra. Esto, sin embargo, como se mencionaba anteriormente, no implica que no haya otro tipo de razones que permitan dirimir la disputa. Muy esquemáticamente, podríamos decir que hay razones conceptuales y razones experimentales a considerar.

con dicha tesis y una argumentación en detalle en favor de ella requeriría el análisis de todas las derivaciones disponibles en la bibliografía, lo que escapa al alcance del presente artículo. Espero, sin embargo, que dicha tesis resulte plausible a la luz de haber presentado las imbricaciones complejas que existen entre la formulación de las reglas operacionales en los cálculos de secuentes y la absorción de reglas estructurales. 
Respecto de las razones que he llamado conceptuales, es importante señalar que una solución a una paradoja requiere, además del formalismo, una interpretación del fenómeno paradójico que vaya de la mano con el abandono de la regla estructural en cuestión en el contexto paradójico. Esto es, además del bloqueo formal de la derivación paradójica, una solución satisfactoria a una paradoja debe traer consigo una respuesta a la siguiente pregunta: ¿por qué tal regla estructural (la que sea que se esté considerando) juega un papel en el razonamiento paradójico? Es decir, ¿por qué esa regla debería fallar en dichos contextos? En otros términos, no es suficiente con mostrar que si fallara entonces la derivación no se seguiría, sino que hay que proveer razones que justifiquen su falla. En este sentido, las soluciones no contractivas y no transitivas tienen cierta ventaja respecto de sus rivales no monotónicas y no reflexivas. Entre otros, Rosenblatt (2019) realiza un compendio de las diversas interpretaciones filosóficas esgrimidas en la bibliografía que justifican el abandono de contracción en el contexto de paradojas. Respecto de las soluciones no transitivas, puede consultarse a Ripley (2012). Además, Ripley (2015b) desarrolla una comparación detallada entre estos dos tipos de soluciones. Entonces, la comparación y la evaluación de los argumentos que justifican la falla de una regla estructural en un contexto paradójico es una de las estrategias para decidir entre distintas soluciones subestructurales.

Por otro lado, se encuentran las razones empíricas o experimentales que podrían aportar evidencia en favor o en contra de una determinada solución subestructural. Como es bien sabido, muchas de las soluciones subestructurales tienen como trasfondo una posición inferencialista. Esto es, muchos de los teóricos subestructurales se han comprometido con la tesis que afirma que el significado de las conectivas lógicas y de la noción de consecuencia lógica (y, por ende, las reglas de los cálculos) está basado en las prácticas inferenciales de los hablantes. Esto ha motivado la utilización de recursos experimentales que testean qué comportamientos tienen los hablantes en contextos paradójicos y cómo modelar formalmente dichos comportamientos (véase, por ejemplo, Ripley, 2016 para una introducción general a la filosofía de la lógica experimental).

Si bien no he ahondado en este trabajo acerca de las paradojas de la vaguedad, las lógicas subestructurales se han propuesto y mostrado eficaces para enfrentar este tipo de razonamientos. En este sentido, se han desarrollado diversas líneas de investigación que explicitan las fallas de monotonía y de transitividad en relación con predicados vagos. Entre otros, podemos mencionar a Cobreros et al. (2016), y también a 
Egré et al. (2013) y Egré y Zehr (2018). Obviamente, el área de filosofía experimental aplicado a lógicas subestructurales está en un estadio incipiente, por lo que no hay evidencia suficiente para destacar una solución subestructural por sobre las demás.

\section{Conclusión}

En este artículo hemos mostrado y enfatizado la algunas veces subestimada relación de dependencia que existe entre las soluciones subestructurales a las paradojas semánticas y las características del cálculo de secuentes en el que se presentan. En particular, nos hemos enfocado en la relación entre la formulación de las reglas operacionales y las reglas estructurales. De este análisis se desprende la necesidad de hacer hincapié en que las reglas estructurales pueden ser absorbidas en las reglas operacionales y que, por ende, la formulación de estas últimas no es inocente en este contexto.

Asimismo, hemos argumentado en contra de una metodología presente en la bibliografía que consiste en tomar como evidencia derivaciones particulares de paradojas en las que una regla estructural no es aplicada explícitamente, a los fines de mostrar que dicha regla no está involucrada en razonamientos paradójicos. Para ilustrar este punto, hemos presentado un cálculo en el que ninguna regla estructural se encuentra explícita en su formulación y que sin embargo es incapaz de lidiar con vocabulario semántico.

El presente análisis no tiene como intención sugerir la ineficacia o falta de pertinencia de las soluciones subestructurales. Muy por el contrario, si bien no hemos argumentado en este artículo en este sentido, creemos con Ripley (2015b) que las soluciones subestructurales están en la ruta correcta dado que enfocan el fenómeno de la paradojicidad en los engranajes del razonamiento, esto es en las reglas que regulan la noción misma de consecuencia lógica. En este sentido, dado que se ha mostrado que para afirmar que una regla no está presente en una derivación de una paradoja, no es suficiente con chequear que dicha regla no se aplique explícitamente, sostenemos que la disputa sobre cuál es (si la hay) la regla responsable o culpable de los razonamientos paradójicos y cuál la teoría subestructural más adecuada para echar luz a estos fenómenos será saldada, en última instancia, en función de las interpretaciones filosóficas brindadas por la teoría sobre el fenómeno y de la adecuación de cada una de ellas a la evidencia empírica pertinente. 


\section{Bibliografía}

Avron, A. (2003). Classical gentzen-type methods in propositional manyvalued logics. In M. Fitting \& E. Orłowska (Eds.), Beyond two: Theory and applications of multiple-valued logic (pp. 117-155). Springer.

Beall, J. (2009). Spandrels of truth. Oxford University Press.

Beall, J., Glanzberg, M., \& Ripley, D. (2016). Liar paradox. In E. N. Zalta (Ed.), The Stanford Encyclopedia of Philosophy (Winter ed.). Stanford University.

Beall, J., Glanzberg, M., \& Ripley, D. (2018). Formal theories of truth. Oxford University Press.

Brady, R. (2006). Universal logic. CSLI Publications.

Caret, C. R., \& Weber, Z. (2015). A note on contraction-free logic for validity. Topoi, 34(1), 63-74.

Cobreros, P., Egré, P., Ripley, D., \& van Rooij, R. (2012). Tolerant, classical, strict. Journal of Philosophical Logic, 41(2), 347-385.

Cobreros, P., Egré, P., Ripley, D., \& van Rooij, R. (2013). Reaching transparent truth. Mind, 122(488), 841-866.

Cobreros, P., Egré, P., Ripley, D., \& van Rooij, R. (2014). Vagueness, truth and permissive consequence. In T. Achourioti, K. Fujimoto, H. Galinon \& J. Martínez-Fernández (Eds.), Unifying the philosophy of truth (pp. 409-430). Springer.

Cobreros, P., Egré, P., Ripley, D., \& van Rooij, R. (2016). Comparing some substructural strategies dealing with vagueness. In J. Carvalho, M. J. Lesot, U. Kaymak, S. Vieira, B. Bouchon-Meunier \& R. Yager (Eds.), Information Processing and Management of Uncertainty in Knowledge-Based Systems. IPMU 2016. Communications in Computer and Information Science, 611 (pp. 161-172). Springer.

Da Ré, B. (2020). Structural weakening and paradoxes. Manuscrito enviado para publicación.

Dutilh Novaes, C., \& French, R. (2018). Paradoxes and structural rules from a dialogical perspective. Philosophical Issues, 28(1), 129-158.

Égré, P., Gardelle V., \& Ripley, D. (2013). Vagueness and order effects in color categorization. Journal of Logic, Language and Information, $22(4), 391-420$

Égré, P., \& Zehr, J. (2018). Are gaps preferred to gluts?: A closer look at borderline contradictions. In E. Castroviejo, G. Weidman Sassoon, \& L. McNally. (Eds.), The semantics of gradability, vagueness, and scale structure: Experimental perspectives (pp. 25-58). Springer. 
Fjellstad, A. (2015). How a semantics for tonk should be. The Review of Symbolic Logic, 8(3), 488-505.

Fjellstad, A. (2016), Naive modus ponens and failure of transitivity. Journal of Philosophical Logic, 45(1), 65-72.

French, R. (2016). Structural reflexivity and the paradoxes of selfreference. Ergo, 3(5), 113-131. http://dx.doi.org/10.3998/ ergo.12405314.0003.005

Hjortland, O. T. (2017). Theories of truth and the maxim of minimal mutilation. Synthese, 1-32.

Mares, E., \& Paoli, F. (2014). Logical consequence and the paradoxes. Journal of Philosophical Logic, 43(2-3), 439-469.

Murzi, J., \& Carrara, M. (2015). Paradox and logical revision: A short introduction. Topoi, 34(1), 7-14.

Murzi, J., \& Shapiro, L. (2015). Validity and truth-preservation. In T. Achourioti, K.Fujimoto, H. Galinon \& J. Martínez-Fernández (Eds.), Unifying the philosophy of truth (pp. 431-459). Springer.

Nicolai, C. \& Rossi, L. (2018). Principles for object-linguistic consequence: From logical to irreflexive. Journal of Philosophical Logic, $47,549-577$.

Ripley, D. (2012). Conservatively extending classical logic with transparent truth. Review of Symbolic Logic, 5(2), 354-378.

Ripley, D. (2014). Review of Replacing truth by Kevin Scharp. Notre Dame Philosophical Reviews. https://ndpr.nd.edu/news/replacingtruth/

Ripley, D. (2015a). Anything goes. Topoi, 34(1), 25-36.

Ripley, D. (2015b). Comparing substructural theories of truth. Ergo, 2(13), 299-328.

Ripley, D. (2016). Experimental philosophical logic. In J. Sytsma \& W. Buckwalter (Eds.), A companion to experimental philosophy (pp. 523-534). Wiley-Blackwell.

Ripley, D. (2018). On the "transitivity" of consequence relations. Journal of Logic and Computation, 28(2), 433-450.

Rosenblatt, L. (2019). Noncontractive classical logic. Notre Dame Journal of Formal Logic, 60(4), 559-585.

Schroeder-Heister, P. (2012). Paradoxes and structural rules. In C. Dutilh Novaes \& O. T. Hjortland (Eds.), Insolubles and consequences: Essays in honour of Stephen Read (pp. 203-211). College Publications.

Schroeder-Heister, P. (2016). Restricting initial sequents: The trade-offs between identity, contraction and cut. In R. Kahle, T. Strahm, \& T. Studer (Eds.), Advances in proof theory (pp. 339-351). Birkhäuser. 
Shapiro, L. (2011). Deflating logical consequence. Philosophical Quarterly, 61, 320-342.

Tarski, A. (1983). Logic, semantics, metamathematics: Papers from 1923 to 1938. Hackett Publishing.

Tennant, N. (2015). A new unified account of truth and paradox. Mind, 124(494), 571-605. https://doi.org/10.1093/mind/fzu179

Weber, Z. (2014). "Naive validity". Philosophical Quarterly, 64(254), 99114.

Weir, A. (2013). A robust non-transitive logic. Topoi, 34(1), 1-9.

Zardini, E. (2011). Truth without contra(di)ction. The Review of Symbolic Logic, 4(4), 498-535.

Zardini, E. (2013). Naive modus ponens. Journal of Philosophical Logic, 42(4), 575-593.

Zardini, E. (2014). Naive truth and naive logical properties. The Review of Symbolic Logic, 7(2), 351-384.

Recibido el 16 de abril de 2020, revisado el 15 de mayo de 2020, aceptado el 25 de mayo de 2020. 